\title{
Effects of the Transverse Curvature on the Statistics of Fully Developed Turbulent Flow in an Annular Pipe
}

\author{
MARCELLO MANNA ${ }^{1, *}$ and ANDREA VACCA ${ }^{2}$ \\ ${ }^{1}$ Dipartimento di Ingegneria Meccanica per l'Energetica, Università di Napoli "Federico II", Via Claudio 21, 80125 Naples, Italy; \\ ${ }^{2}$ Dipartimento di Ingegneria Civile, Seconda Università di Napoli, Aversa, Italy
}

\begin{abstract}
The fully developed turbulent flow in an annular pipe with radius ratio of $1 / 2$ is investigated for Reynolds numbers based on hydraulic diameter and bulk velocity ranging from 12000 to 46000 . The study is carried out processing data obtained from wall resolved large eddy simulations employing the dynamic subgrid scale eddy viscosity model of Germano et al. (1991) as modified by Lilly (1992). Scaling in wall variables shows that the turbulence intensities in the inner radius region are systematically less than the corresponding plane channel data, while the differences in the outer radius region reduce considerably. Because of the transverse curvature the total shear stress deviates from the linear distribution by as much as $12 \%$ in the inner wall region at $y^{+}=100$. The curvature effects decrease as the Reynolds number is increased. Thus in the low Reynolds number range similarity arguments have to be considered with augmented caution with respect to the plane channel case.
\end{abstract}

Keywords: Annulus; Turbulent flows; Curvature effects; Wall scaling; LES

The scientific community has long been at the forefront of the quest to increase the knowledge of turbulence so that a more comprehensive support to the development of fluid dynamic processes of industrial relevance could be attained. Annular conduits are extremely frequently used in axial turbomachines both as inlet and outlet piping systems, but their application is very often encountered in many other engineering devices such as heat exchangers, gas cooled nuclear reactors, combustion chambers, and drilling wells to

\footnotetext{
Received 7 February 2001; in final form 27 March 2001

* Corresponding author. Tel.: + 390817683287 , Fax: + 3908123941

65. E-mail: manna@cds.unina.it
}

name a few. Unlike circular pipe and parallel plate flows which have represented the test benches to explore very many fundamental issues of wall bounded turbulence, and have thus been thoroughly investigated, annular flow has received little attention. Yet pipe and plane channel flows are both limiting cases of the annular flow.

Because of the transverse curvature mean velocity profiles, shear stresses and turbulence intensities exhibit asymmetries which become more pronounced as the radius ratio decreases. Convex curvature effects of wall bounded turbulent flows were investigated by Neves et al. (1994) and Neves and Moin (1994); they analyzed data obtained from direct numerical simulation of a model problem at low Reynolds number $(R e=3300)$, approximating axial boundary layer flow over long cylinders characterized by a ratio of the boundary layer thickness to the cylinder radius of 5 and 11 . The most comprehensive experimental studies of fully developed annular turbulent flows are due to Brighton and Jones (1964) and Reheme (1974); while the former investigated several geometries (radius ratios between 0.0625 and 0.562 ) at fairly high Reynolds number (viz. $46000 \leq R e \leq 327000$ ), the latter concentrated on thin rods (radius ratios between 0.02 and 0.1 ) at moderate Reynolds numbers (i.e., $R e \leq 91000$ ). More recently some data have been provided by Nouri et al. (1993) for a single geometry (radius ratio of $1 / 2$ ) and two Reynolds numbers (i.e., $R e=8900$ and 26600 ). Both studies, however, do not have enough resolution to confidently allow for the near wall behavior analysis.

The present investigation provides detailed information on the dependence of the most relevant turbulent quantities with the Reynolds number, based on the bulk velocity $\left(\bar{u}_{b}\right)$ and the hydraulic diameter $(2 s)$, in the range $12000 \leq R e \leq 46000$, for a single configuration characterized by a radius ratio of $1 / 2$. The study is conducted analyzing data obtained from very well resolved large eddy simulation (LES) carried out with a fully spectral Navier-Stokes solver (Manna \& Vacca, 1999). The 
filtered equations are closed with the dynamic sub-grid scale model of Germano et al. (1991), modified by Lilly (1992). Attention is focused on the deviation from the linear distribution of the shear stress with the Reynolds number. The numerical method, the problem set-up and the sub-grid scale model is described in the next section. All data are presented and discussed in the third section. In the appendix a grid refinement study is carried out to demonstrate the quality of the data.

\section{PROBLEM FORMULATION}

The operation of filtering the Navier-Stokes equations leads to large scale motion equations in terms of the resolved (denoted with a tilde) components, $\tilde{\mathbf{u}}$ and $\tilde{p}$ :

$$
\begin{gathered}
\frac{\partial \tilde{\mathbf{u}}}{\partial t}+\nabla \cdot \tilde{\mathbf{u}} \tilde{\mathbf{u}}=-\frac{1}{\rho} \nabla \tilde{p}+\frac{1}{R e} \nabla \cdot \tilde{\mathbf{S}}-\frac{1}{\rho} \nabla \cdot \tau, \\
\nabla \cdot \tilde{\mathbf{u}}=0
\end{gathered}
$$

in which $\tilde{\mathbf{S}}$ and $\tau=\rho(\tilde{\mathbf{u}} \tilde{\mathbf{u}}-\widehat{\mathbf{u u}})$ are the resolved rate of strain and subgrid-scale stress tensors. In the present paper we have added the isotropic part of $\boldsymbol{\tau}$ to the pressure term and parametrized the anisotropic part of the subgrid-stress tensor by an eddy viscosity assumption

$$
\tau-\frac{1}{3} \operatorname{Tr}(\tau)=-2 \mu_{T} \tilde{\mathbf{S}}=-2 C(r, t) \rho \Delta|\tilde{\mathbf{S}}| \tilde{\mathbf{S}}
$$

with $|\tilde{\mathbf{S}}|=\sqrt{\operatorname{Tr}\left(2 \tilde{\mathbf{S}}^{2}\right)}, \operatorname{Tr}$ the trace operator and $\Delta$ the length associated with the grid scale filter. Following Lilly's modification (Lilly (1992)) to the Germano model (Germano et al. (1991)) and introducing a second coarser spatial (test) filter characterized by a width $\hat{\Delta}$, the scalar function $C(r, t)$ is given by

$$
C(r, t)=-\frac{1}{2} \frac{\langle\operatorname{Tr}(\mathbf{L M})\rangle}{\langle\operatorname{Tr}(\mathbf{M M})\rangle}
$$

where $\langle\cdot\rangle$ denotes averaging in both azimuthal and axial directions and

$$
\mathbf{L}=\widehat{\tilde{\mathbf{u}}}-\widehat{\tilde{\mathbf{u}}} \widehat{\tilde{\mathbf{u}}}, \quad \mathbf{M}=\hat{\Delta}^{2}|\hat{\tilde{\mathbf{S}}}| \hat{\tilde{\mathbf{S}}}-\Delta^{2}|\widehat{\widetilde{\mathbf{S}}}| \widehat{\tilde{\mathbf{S}}}
$$

Sharp Fourier cutoff is used as both grid and test filter in streamwise and azimuthal directions, while no test filtering is performed in the radial direction. The test filter width is such that $\hat{\Delta} / \Delta=2$ (Piomelli, 1993).
The governing Eqs. [1] and [2] have been solved using the initial value solver developed and validated in Manna and Vacca (1999). The numerical method relies on a spectral Chebyshev multi-domain algorithm for the inhomogeneous (radial) direction and Fourier decomposition for the homogeneous (streamwise and azimuthal) ones. Time integration is carried out with a Crank-Nicolson scheme for the viscous terms. The non-linear convective terms, in skewsymmetric form, are treated explicitly with an Adams Bashforth scheme. Periodic boundary conditions are applied in streamwise and azimuthal directions, and no-slip boundary conditions at the solid walls.

\section{RESULTS}

The sizes of the computational domain in the streamwise, wall normal and azimuthal directions were $8 s \times s \times 2 \pi / 4$, for the $R e=12000$ case, $4 s \times s \times 2 \pi / 8$, for the $R e=29000$ and $3.4 s \times s \times 2 \pi / 12$, for the $R e=46000$. The lengths in the streamwise and azimuthal directions are $L_{z}=2940$, $3170,4000, L_{\theta}=1000$ wall units for the three Reynolds numbers. The grid resolution is detailed in the appendix were a grid refinement study is carried out. Both spatial averaging in the homogeneous $z$ and $\theta$ directions, and time averaging are applied to collect the statistical data. The low Reynolds number field was used as initial solution for the two higher Reynolds number cases; the low Reynolds case was initiated from a randomly generated field with a laminar mean velocity distribution.

From here onwards we shall denote with overline the quantities which have been averaged over $z, \theta$ and $t$, and with prime the perturbation from their averages. Finally, we shall drop the tilde in the interest of simplicity of notations.

\section{Global Parameters}

For a fully developed, axisymmetric steady flow in annular geometry, the integration of momentum equation over the gap gives:

$$
-\frac{d p}{d z}=\frac{2 \tau_{w, m}}{s}
$$

where $\tau_{w, m}$ is the shear stress averaged over the inner and outer walls $\tau_{w, m}=\left(\tau_{w, i} r_{i}+\tau_{w, o} r_{o}\right) /\left(r_{i}+r_{o}\right)$.

In Table I we give, for all Reynolds numbers, the friction factor deduced from the Blasius law $C_{f}^{B}=$ $0.079 R e^{-1 / 4}$, the calculated ones, the percentage changes along with the ratios between the inner and outer wall shear stresses. The computed skin friction coefficients are in very close agreement with the predicted ones with 
TABLE I Friction factors $C_{f}=2 \tau_{w, m} / \rho \bar{u}_{b}^{2}$, and ratios of the wall shear stresses

\begin{tabular}{lcccc}
\hline$R e_{b}$ & $C_{f}^{B} \times 10^{3}$ & $C_{f} \times 10^{3}$ & $\left(C_{f}-C_{f}^{B}\right) / C_{f}^{B}$ & $\tau_{w, i} / \tau_{w, o}$ \\
\hline 12000 & 7.55 & 7.49 & $0.80 \%$ & 1.13 \\
29000 & 6.05 & 5.96 & $1.49 \%$ & 1.09 \\
46000 & 5.39 & 5.25 & $2.66 \%$ & 1.10 \\
\hline
\end{tabular}

a little dependence on the Reynolds number; similarly the ratios of the wall shear stresses show a moderate Reynolds dependence and a clear reduction with respect to the corresponding laminar value $\left(\tau_{w, i} / \tau_{w, o}=1.27\right)$. The prediction of the wall stress is considered reliable mainly because the grid size in the spanwise direction $8 \leq$ $(r \Delta \theta)^{+} \leq 16$ is sufficient to resolve the most important wall layer structures, viz. the low speed streaks. In
Figure 1 we show the contours of the instantaneous radial vorticity $\omega_{r}^{\prime}=1 / r \partial u_{z}^{\prime} / \partial \theta-\partial u_{\theta}^{\prime} / \partial z$ scaled by $u_{\tau}^{2} / \nu$ in the $(\theta, z)$-plane at $y^{+}=10$, for both walls. The thin and elongated layers of $\omega_{r}^{\prime}$ represent high and low speed streaks, the coherent structures mainly contributing to build the $\overline{u_{z}^{\prime} u_{r}^{\prime}}$ Reynolds stress. Modifications of the radial distribution of $\overline{u_{z}^{\prime} u_{r}^{\prime}}$ can usually be interpreted in terms of changes in the bursting process, a fact that can be quantified through the quadrant analysis (Wallace et al. (1972)). Here we do not concentrate on the statistical characterization of the streaks during the sweep and ejection events (like in Guezennec et al., 1989); rather we simply wish to stress that these events are properly resolved, as evident from the instantaneous contour of the axial velocity in the $(r, \theta)$-plane presented in Figure 2. The graphs, in inner coordinates, depict

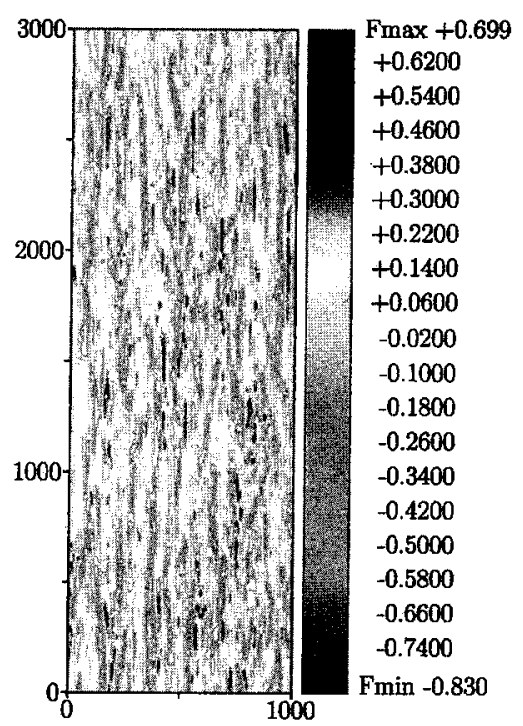

FIGURE 1 Instantaneous contour values of $\omega_{r}^{\prime}$ scaled by $u_{\tau}^{2} / \nu$ in the $(\theta, z)$-plane in wall units at $y^{+}=10$; contour level $\Delta=0.08$. Lower wall, left, upper wall, right (See Colour Plate at back of issue.).
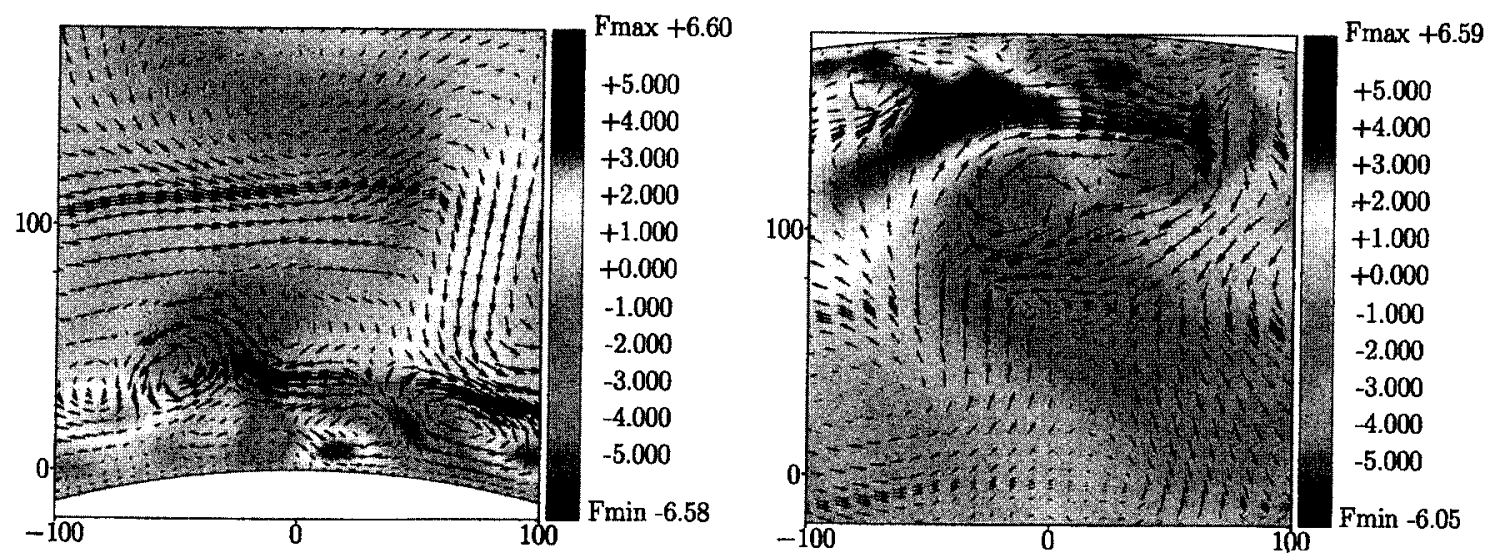

FIGURE 2 Instantaneous contour values of $u_{z}^{\prime}$ scaled by $u_{\tau}$ in the $(r, \theta)$-plane in wall units; contour level $\Delta=0.5$. Lower wall, left, upper wall, right (See Colour Plate at back of issue.). 
typical low speed wall-outward fluid flow (ejections, blue areas) and high speed wall-ward flow (sweep, red areas).

The present results together with the experimental data of Brighton and Jones (1964) allow to cover a significant Reynolds number range (practically spanning two orders of magnitude). The weak variation of the $\tau_{w, i} / \tau_{w, o}$ ratio with $R e$ is of relevance for the shear stress distribution across the channel and is addressed in the Reynolds stress subsection.

\section{Mean Velocity}

In Figure 3 we present the mean axial velocity profile normalized by the maximum velocity across the duct in outer coordinates, for all Reynolds numbers. The computations at $R e=29000$ compare rather well with the experimental data of Nouri et al. (1993) obtained at a slightly different Reynolds number $(R e=26600)$. The Reynolds number effects on the velocity distributions in terms of change of the location of the maximum velocity is negligible. In Figure 4 we show the mean velocity profiles in inner coordinates, each of which has been normalized by the corresponding friction velocity $u_{\tau}=\sqrt{\tau_{w} / \rho}$. More significant is the deviation of the inner profile from the logarithmic distribution $\left(u^{+}=1 / \kappa \ln \left(y^{+}\right)+B\right.$, with a von Kármán constant of $\kappa=0.41$ and an additive constant $B=5.5$ ), compared to the outer one. As the Reynolds number is increased the logarithmic layer becomes more neat, and the two higher Re profiles collapse in a fairly large interval. Also their intercept with the logarithmic distribution is nearly coincident. Note that all simulations show a very well resolved inner layer. Whether the

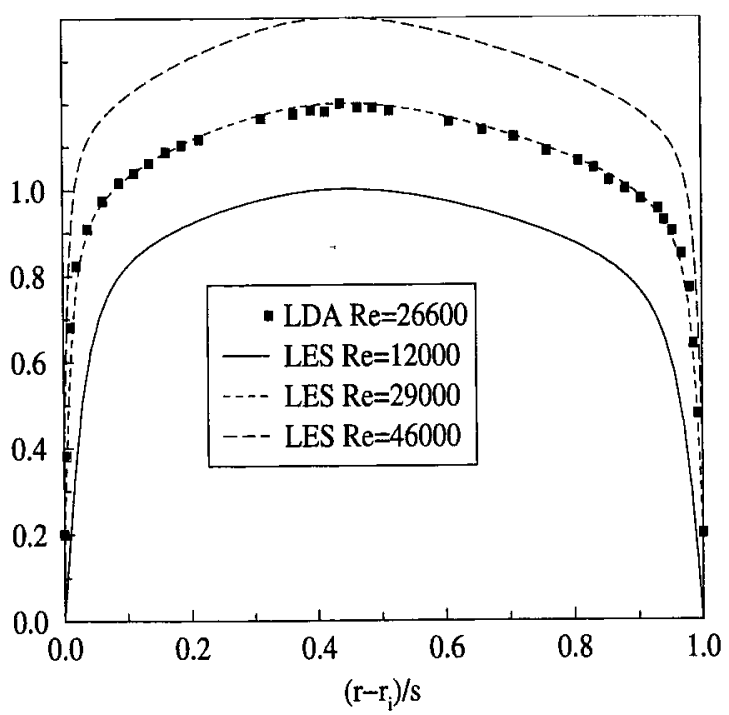

FIGURE 3 Axial mean velocity profile, scaled by $\bar{u}_{c}$, in outer coordinates; - experiments from Nouri et al. (1993).

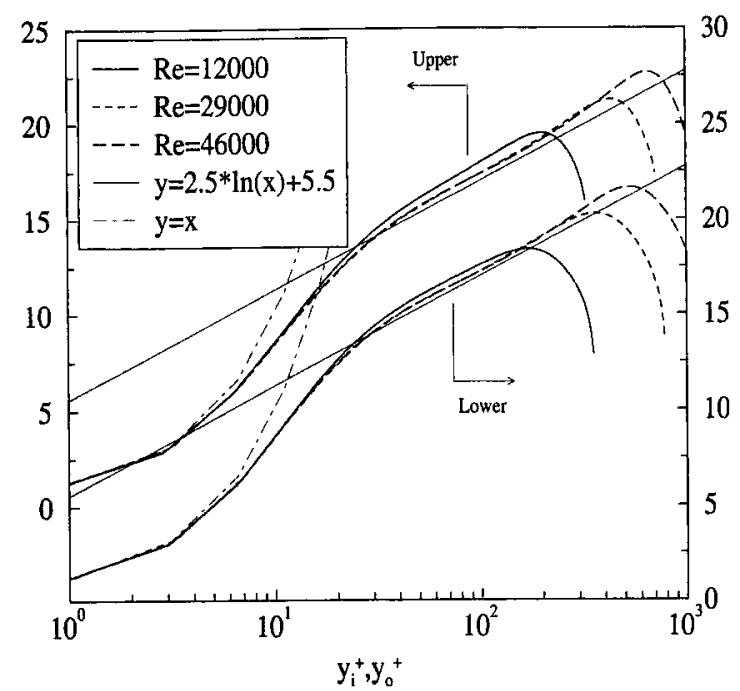

FIGURE 4 Axial mean velocity profile in inner coordinates. Lower wall right, upper wall left.

similarity in the overlap region is incomplete because the $\kappa$ and $B$ constants are $R e$ dependent is still an open question; however, we wish to point out that transverse curvature has a non negligible effect on the log law, and thus, similarity arguments have to be treated with caution at least in the low Reynolds number range.

\section{Reynolds Stresses}

In Figure 5 we show the radial distribution of the turbulence intensities and the shear stress in outer coordinates for a single Reynolds number for which experimental data are

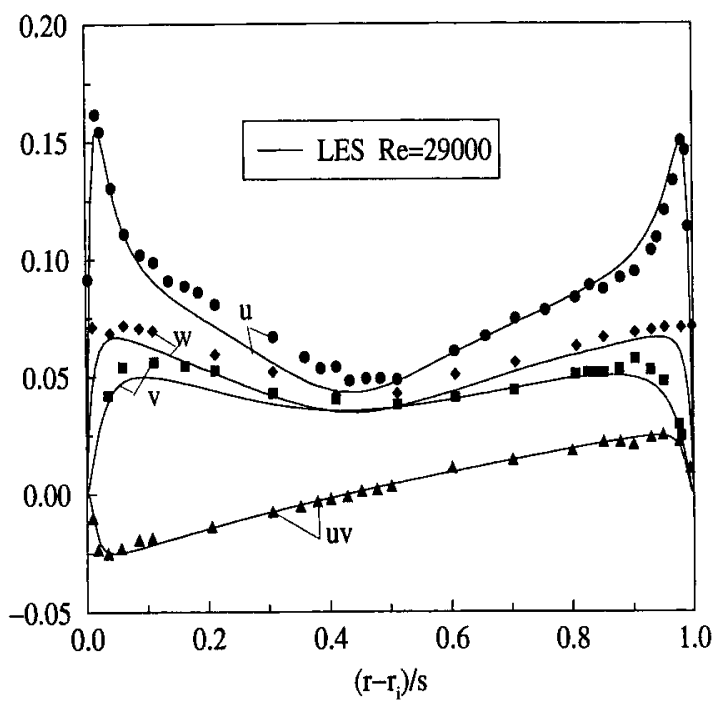

FIGURE 5 Reynolds stresses in outer coordinates; symbols denote experiments from Nouri et al. (1993) at $R e=26600 . \bullet,{\overline{u_{z}^{\prime 2}}}^{1 / 2} / \bar{u}_{b}$; $\overline{u_{r}^{\prime 2}}{ }^{1 / 2} / \bar{u}_{b} ; \diamond,{\overline{u_{\theta}^{\prime 2}}}^{1 / 2} / \bar{u}_{b} ; \mathbf{\Lambda}, \overline{u_{z}^{\prime} u_{r}^{\prime}} / \bar{u}_{b}^{2}$. 
available (Nouri et al., 1993). All data were normalized by the bulk velocity. The anisotropy among the stresses remains pronounced at a large distance from both walls; the location of all minima basically coincide with that of maximum mean velocity and zero shear stress. The accordance between the predicted and measured values is good all over the annulus gap. To highlight the differences between the inner and outer near wall distributions of the turbulence intensities we present in Figure 6 the data separately for each wall, and compare them with the plane channel DNS data of Moser et al. (1999). All quantities have been normalized by the corresponding friction velocity. There is some Reynolds number effects using inner variables scaling; we are confident that this result is not an artifact of the sub-grid scale model, since the computations were carried out with identical filter widths in wall units, so that the ratio of the unresolved to the wall shear stress remains, roughly speaking, a constant fraction of the total stress. In other words the contribution of the SGS term is small and constant as the Reynolds number is increased. With reference to the inner wall distributions we observe that all turbulence intensities are systematically less than the corresponding plane channel counterparts across the whole annulus gap. As concerns the outer wall the differences with the plane channel data reduce considerably, especially for the radial and azimuthal components.

Figure 7 shows that the turbulent shear stresses compare well with the plane channel of Moser et al. (1999) in the near wall region $\left(y_{o}^{+} \leq 100\right)$ of the outer radius; the collapse is outstanding for $R e=46000$. Conversely in the inner radius region the scatter is more pronounced, but it improves with the Reynolds number. The difference in the turbulent stress distribution, both in terms of maximum strength and location, is significant. With reference to the inner wall, for instance, the locations of maximum turbulent stress are $\sim 31$ (for $R e=12000$ ), $\sim 38$ (for
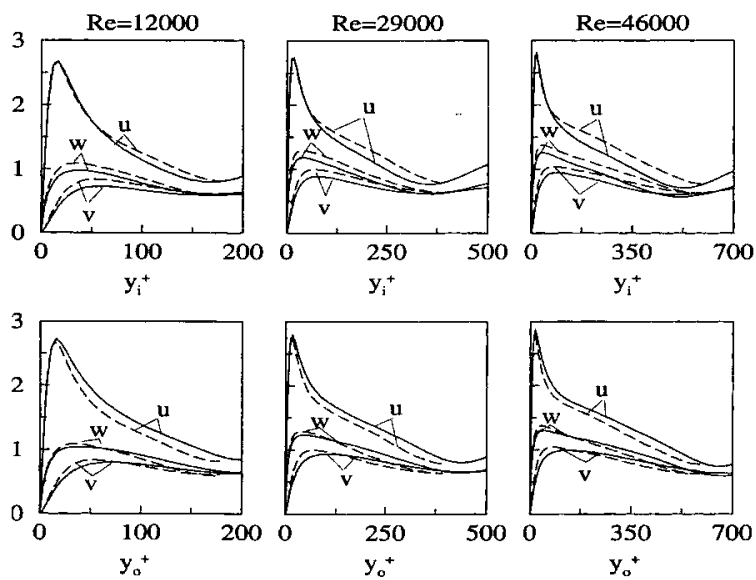

FIGURE 6 Turbulence intensities in inner coordinates; dashed lines denote plane channel DNS data from Moser et al. (1999).
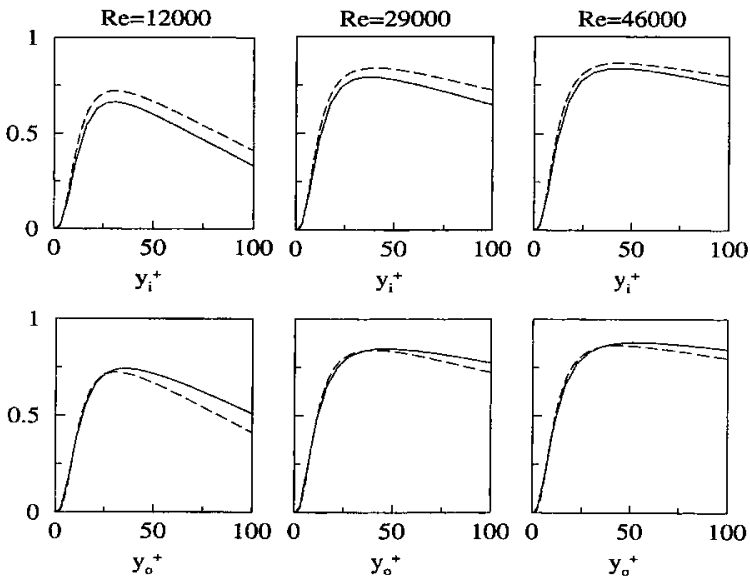

FIGURE 7 Turbulent shear stress (resolved +SGS) in the wall region; dashed lines denote plane channel DNS data from Moser et al. (1999).

$R e=29000$ ) and $\sim 44$ (for $R e=46000$ ); the corresponding values are $\sim 0.66, \sim 0.79$ and $\sim 0.84$.

In Figure 8 we present, in inner coordinates, the computed viscous stress, the resolved and resolved + SGS turbulent stress, along with the total ones, for all Reynolds numbers. Note that the magnitude of the contribution of the subgrid model to the turbulent shear stress is at most a few units percent. This observation confirms the high quality of the present data. The comparison between the total shear stresses at the inner and outer walls shows the substantial difference in the position of the zero points $y_{0, i}^{+}, y_{0, o}^{+}: \operatorname{Re}=12000: y_{0, i}^{+} \sim 170, y_{0, o}^{+} \sim 200 ; \quad \operatorname{Re}=29000:$ $y_{0, i}^{+} \sim 355, y_{0, o}^{+} \sim 440 ; \operatorname{Re}=46000: y_{0, i}^{+} \sim 520, y_{0, o}^{+} \sim 658$. The deviation from the linearity of the total shear stress clearly appears from the figure for all Reynolds numbers. This behavior is consistent with the theoretical radial distribution of the total shear stress that can be easily deduced from the integration across the channel gap of the
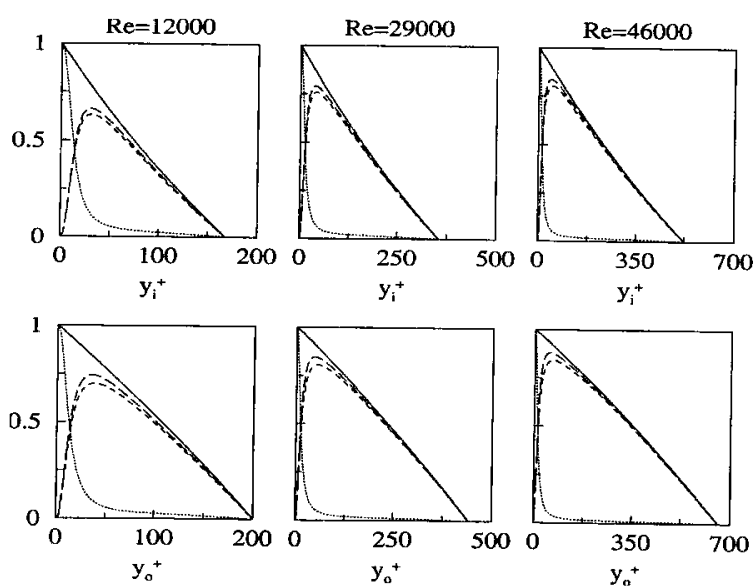

FIGURE 8 Shear stress in inner coordinates; ..... viscous, - - turbulent (resolved), - - - turbulent (resolved + SGS), - total. 
streamwise component of the momentum equation. Evaluating the integration constant at $r=r_{i}$ the radial distribution reads

$$
\begin{aligned}
\frac{d \bar{u}^{+}}{d y_{i}^{+}}-\overline{u_{z}^{\prime} u_{r}^{+}}= & f\left(y_{i}^{+}, \operatorname{Re} \tau, \frac{s}{r_{i}}\right) \\
& -\frac{y_{i}^{+}}{\operatorname{Re} e_{\tau}} g\left(y_{i}^{+}, R e_{\tau}, \frac{s}{r_{i}}, \frac{\tau_{w, n}}{\tau_{w, i}}\right),
\end{aligned}
$$

where the functions $f$ and $g$ are:

$$
\begin{aligned}
f\left(y_{i}^{+}, \operatorname{Re}_{\tau}, \frac{s}{r_{i}}\right)= & \frac{1}{1+\left(y_{i}^{+} / 2 \operatorname{Re}_{\tau}\right)\left(s / r_{i}\right)}, \\
& g\left(y_{i}^{+}, \operatorname{Re}_{\tau}, \frac{s}{r_{i}}, \frac{\tau_{w, m}}{\tau_{w, i}}\right) \\
= & \frac{1+\left(y_{i}^{+} / 4 \operatorname{Re}_{\tau}\right)\left(s / r_{i}\right)}{1+\left(y_{i}^{+} / 2 \operatorname{Re} e_{\tau}\right)\left(s / r_{i}\right)} \frac{\tau_{w, m}}{\tau_{w, i}}
\end{aligned}
$$

with $R e_{\tau}$ based on the average friction velocity and the hydraulic radius $\left(r_{h}=s / 2\right)$. A similar expression can be deduced with reference to the outer wall. From the definitions [8] it follows that non linearities affect both functions $f$ and $g$. For large values of $\operatorname{Re}_{\tau}\left(\operatorname{Re}_{\tau} \rightarrow \infty\right)$, it is easy to verify that $f \rightarrow 1$ and $g \rightarrow \tau_{w, m} / \tau_{w, i}$, and the linear distribution is recovered. In the present work the curvature parameter $s / r_{i}$ is obviously fixed, while the ratio $\tau_{w, m} / \tau_{w, i}$, and thus $\tau_{w, i} / \tau_{w, o}$ is seen to change very modestly with $R e$ (see Table I), so that the deviations from the linear distribution have to be attributed to the Reynolds number effects. To give a quantitative evidence of the above claims we present in Figure 9 the effects of $R e_{\tau}$ onto the deviations of the total stress distribution from the linear one, for both

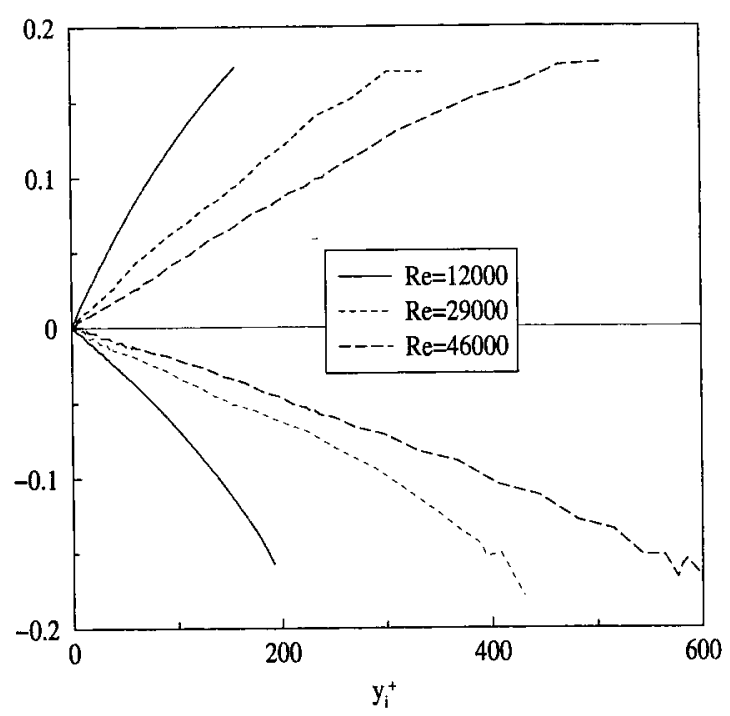

FIGURE 9 Deviation of the total shear stress from the linear behavior; lower wall, top, upper wall, bottom. inner and outer walls, non dimensionalized by the corresponding linear value. The transverse curvature effects are definitely more pronounced in the inner wall region; at $y^{+}=100$ the drift from the linear distribution is $7 \%$ in the outer wall and $12 \%$ in the inner one, at $R e=12000$. The differences reduce as the Reynolds number is increased, although they are far from being negligible even at the highest Reynolds.

As already anticipated the observed differences in the turbulent stress distributions of both walls can be interpreted in terms of coherent structures modifications. Joint probability density functions of the streamwise and azimuthal velocities, and spanwise two points correlations (results not shown herein) corroborate the above scenario providing evidence that the transverse curvature has a deceptive effect onto the number and strength of the Reynolds stress producing events, a fact that also appears from the analysis of the instantaneous realizations presented in Figure 1. Note that, the graphs being in wall units, the radial vorticity distributions of the two wall layers are directly comparable.

\section{CONCLUSIONS}

Large eddy simulation of a fully developed turbulent annular pipe with a radius ratio of $1 / 2$ have been carried out with a spectral multi-domain Navier-Stokes solver. The study addressed the effects of the transverse curvature upon the main turbulence quantities in a Reynolds number range of $12000 \leq R e \leq 46000$. The mean velocity distributions exhibit a fairly neat logarithmic layer for the two higher Reynolds numbers, while at $R e=12000$ the inner and outer profiles differ considerably. Scaling in wall variables shows that the turbulence intensities in the inner radius region are systematically less than the corresponding plane channel data, while the differences in the outer radius region are definitely smaller. The total shear stress deviates from the linear distribution by as much as $12 \%$ in the inner wall region at $y^{+}=100$, although the differences diminish as the Reynolds number is increased. All data indicate that in the low Reynolds number range similarity arguments have to be considered with augmented caution with respect to the plane channel case.

\section{NOMENCLATURE}

\section{Dimensional Quantities}

$t$ time coordinate, time

$z$ axial coordinate, length

$r$ radial coordinate, length

$y \quad$ distance from the wall, length 


\section{Non-dimensional Quantities}

Re Reynolds number

$C_{f} \quad$ friction coefficient

$\kappa \quad$ von Kármán constant

$k$ wave number

\section{Subscripts}

$i \quad$ inner cylinder radius, lower wall

$o \quad$ outer cylinder radius, upper wall

$m$ mean cylinder radius

$b$ bulk, area averaged value

$c$ centerline value

$w \quad$ wall value

0 radial position of zero value

\section{Superscripts}

$+\quad$ wall coordinates

, fluctuating component

$B \quad$ Blasius value

\section{REFERENCES}

Brighton, J. A. and Jones, J. B. (1964) Fully Developed Turbulent Flow in Annuli, Journal of Basic Engineering, 2, 835-844.

Germano, M., Piomelli, U., Moin, P. and Cabot, W. H. (1991) A Dynamic Subgrid Scale Eddy Viscosity Model, Physics of Fluids, 3, 1760-1767.

Guezennec, Y. G., Piomelli, U. and Kim, J. (1989) On the Shape and Dynamics of Wall Structures in Turbulent Channel Flow, Physics of Fluids, 1(4), 764-766.

Lilly, D. K. (1992) A Proposed Modification of the Germano SubgridScale Closure Method, Physics of Fluids, 4(3), 633-635.

Manna, M. and Vacca, A. (1999) An Efficient Method for the Solution of the Incompressible Navier-Stokes Equations in Cylindrical Geometries, Journal of Computational Physics, 151, 563-584.
Moser, R., Kim, J. and Mansour, N. (1999) Direct Numerical Simulation of Turbulent Channel flow up $R e=590$, Physics of Fluids, 11(4), $1938-1944$.

Neves, J., Moin, P. and Moser, R. (1994) Effects of Convex Transverse Curvature on Wall Bounded Turbulence. Part 1. The Velocity and Vorticity, Journal of Fluid Mechanics, 272, 349-381.

Neves, J. and Moin, P. (1994) Effects of Convex Transverse Curvature on Wall Bounded Turbulence. Part 2. The Pressure Fluctuations, Journal of Fluid Mechanics, 272, 383-406.

Nouri, J. M., Umur, H. and Whitelaw, J. H. (1993) Flow of Newtonian and non-Newtonian Fluids in Concentric and Eccentric Annuli, Journal of Fluid Mechanics, 253, 617-641.

Piomelli, U. (1993) High Reynolds Number Calculations Using the Dynamic Subgrid Scale Stress Model, Physics of Fluids, 5(6), $1484-1490$.

Reheme, K. (1974) Turbulent Flow in Smooth Concentric Annuli with Small Radius Ratios, Journal of Fluid Mechanics, 64, 263-287.

Wallace, J. M., Eckelmann, H. and Brodkey, R. S. (1972) The Wall Region in Turbulent Shear Flow, Journal of Fluid Mechanics, 54, $39-48$.

\section{APPENDIX: ACCURACY CHECK}

The accuracy of the whole set of data is tested performing a grid refinement study increasing the number of modes in the homogeneous and inhomogeneous directions separately. Statistics for the coarse and fine grids were collected over identical averaging times, so that the sample size plays no role in the present discussion. The data are obtained processing 50 nearly independent fields separated in time by $0.1 t^{+}$where $t^{+}=t u_{\tau, m} /(2 s)$. The time steps of the simulations are $\Delta t^{+}=0.31 \times 10^{-3}, 0.14 \times 10^{-3}, 0.05 \times$ $10^{-3}$ for the three Reynolds numbers respectively. When the grid was refined the time step was reduced to $\Delta t^{+}=$ $0.15 \times 10^{-3}$.

Table II shows the main computational parameters in inner coordinates; the study pertains to the lower Reynolds number case only. However, since the box size in wall units is kept constant when the Reynolds number is increased, we claim that the outcome of the present discussion is applicable to the whole set of calculation presented herein. To have an idea of how fine is the grid, that is, how well we describe the turbulent flow features with respect to the dissipative scale $\ell_{d}$, we must compare the mean grid size $\Delta=(\Delta z \Delta r r \Delta \theta)^{1 / 3}$ with the Kolmogorov length scale $\eta=\left(\nu^{3} / \bar{\varepsilon}\right)^{1 / 4}$. Since we know from experiments that the wave number range where energy dissipation takes place is $0.1<2 \pi \eta / \ell_{d}^{+}<1.0$, if $\Delta^{+}<\ell_{d}^{+} / 2$ the simulation may be considered direct; in a LES context this condition is obviously relaxed, since the small scales are modeled. However, it is interesting to compare the $\pi \eta$ bound with the mean grid size $\Delta$, precisely to have an idea where the cut-off stands. The comparison is carried out in inner coordinates in Figure 10 where shown is also the Kolmogorov scale of the plane channel flow DNS of Moser et al. (1999). The averaged dissipation $\bar{\varepsilon}$ needed to evaluate $\eta$ is obtained from the super-grid Reynolds stress budgets (results not shown). Note the excellent agreement 
TABLE II Characteristics of the reference and refined grids

\begin{tabular}{lcccc}
\hline$R e$ & Grid size & $\Delta z^{+}$ & $\Delta r^{+}$ & $(r \Delta \theta)^{+}$ \\
\hline 12000 & $6 \times(64 \times 12 \times 72)$ & 46 & $0.7 \leq \Delta r^{+} \leq 10.4$ & $8.0 \leq(r \Delta \theta)^{+} \leq 16.0$ \\
29000 & $6 \times(64 \times 12 \times 72)$ & 50 & $0.8 \leq \Delta r^{+} \leq 33.8$ & $8.7 \leq(r \Delta \theta)^{+} \leq 17.3$ \\
46000 & $6 \times(64 \times 15 \times 72)$ & 64 & $0.7 \leq \Delta r^{+} \leq 39.5$ & $8.6 \leq(r \Delta \theta)^{+} \leq 17.1$ \\
12000 & $6 \times(96 \times 12 \times 108)$ & 32 & $0.8 \leq \Delta r^{+} \leq 10.7$ & $5.5 \leq(r \Delta \theta)^{+} \leq 10.9$ \\
12000 & $6 \times(64 \times 16 \times 72)$ & 46 & $0.4 \leq \Delta r^{+} \leq 7.7$ & $8.0 \leq(r \Delta \theta)^{+} \leq 16.0$ \\
\hline
\end{tabular}

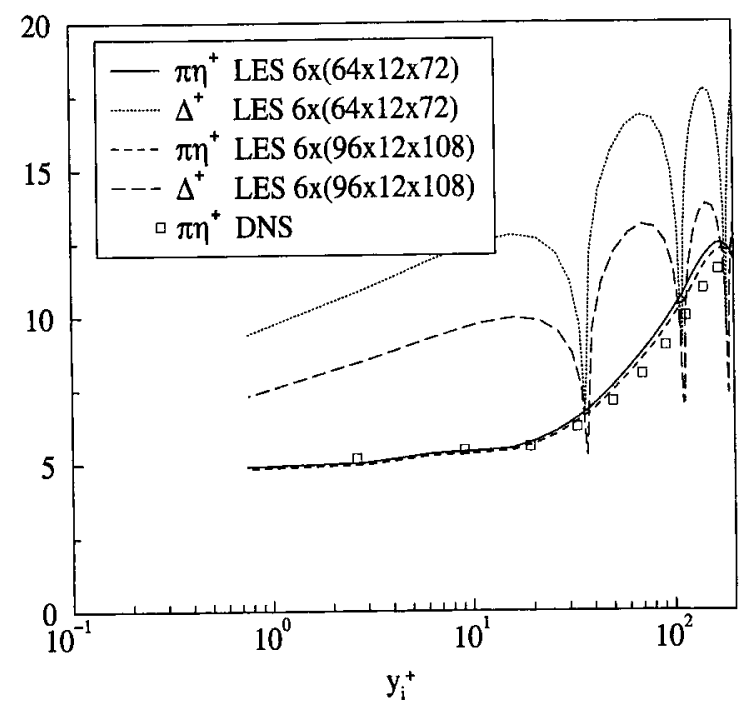

FIGURE $10 \pi \eta$ bound and mean mesh size in inner coordinates (lower wall); DNS data from Moser et al. (1999).

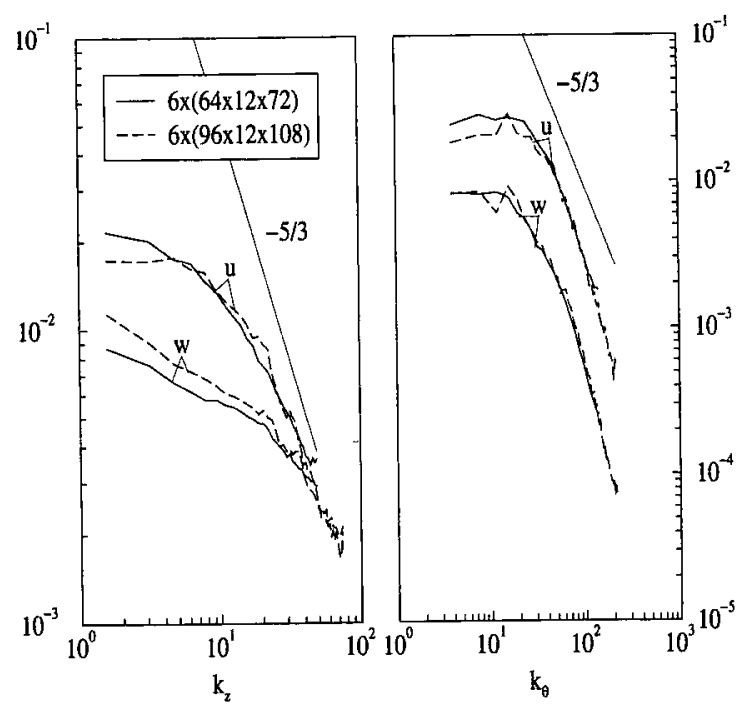

FIGURE 11 One dimensional energy spectra in $z$ (left) and $\theta$ (right) directions.

of the computed $\eta$ distribution with the DNS of Moser et al. (1999). The figure clearly shows that even the coarse grid simulation enjoys a remarkable resolution since the mean grid size is practically everywhere less than two times the
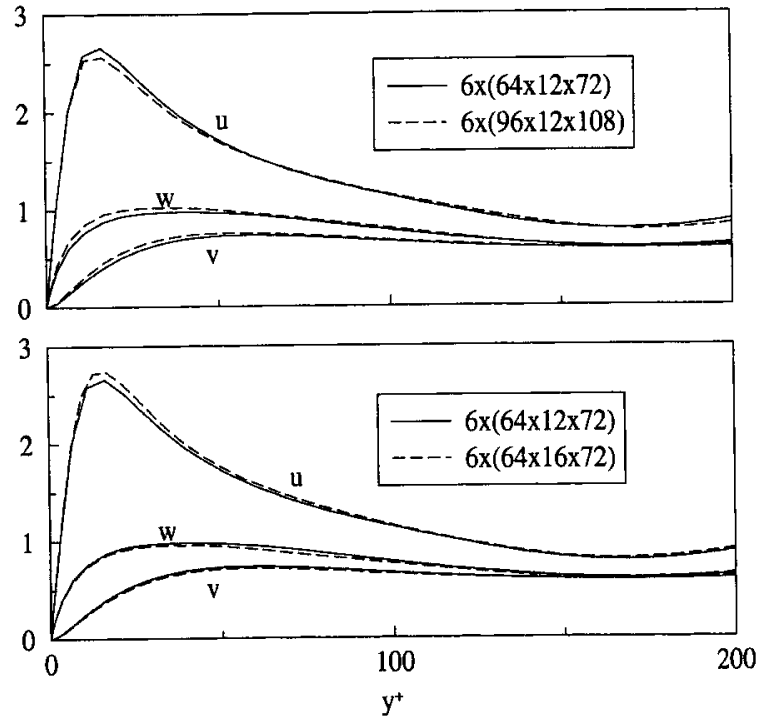

FIGURE 12 Turbulence intensities in inner coordinates; refinement in $z-\theta$ (top) and $r$ (bottom).

dissipative length scale. Thus almost all relevant turbulent scales have been resolved. In Figure 11 we present the one dimensional energy spectra of the axial and azimuthal velocity components, normalized with the friction velocity, in wavenumber space $\left(k_{z}=2 \pi k / L_{z}\right.$ and $\left.k_{\theta}=2 \pi k / L_{\theta}\right)$ at $y^{+}=70$. The drop in the energy is definitely more pronounced in the azimuthal direction compared to the axial one because of the smaller mesh spacing $\left(r \Delta \theta^{+} \approx \Delta z^{+} / 3\right)$. There is essentially no energy pile up near the cutoff wave numbers, but more important the spectra remains practically unchanged when the resolution is increased by $50 \%$ in the homogeneous directions.

Finally in Figure 12 we compare the turbulence intensities as obtained by refining the grid both in the inhomogeneous $(r)$ and homogeneous $(z, \theta)$ directions. The differences are comfortably small. From the foregoing we conclude that the overall grid resolution is such that the percentage of energy contained in the subgrid scales is an insignificant fraction of the total; the energy spectra and the Kolmogorov estimate confirm the fact that both the grid and test filter of the sub-grid model are very near to the dissipative range. Thus we have extreme confidence in the quality of the collected statistics. 

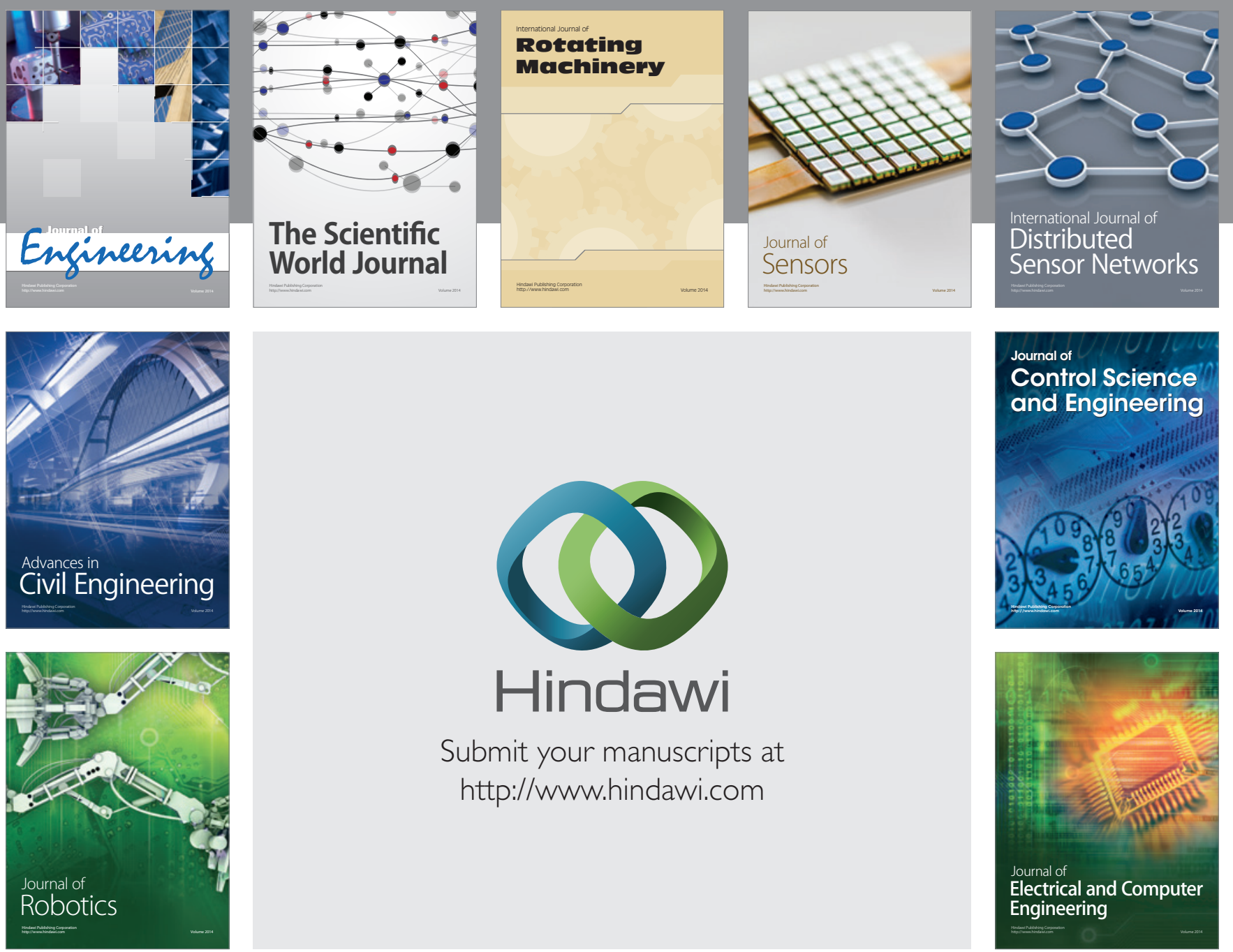

Submit your manuscripts at

http://www.hindawi.com
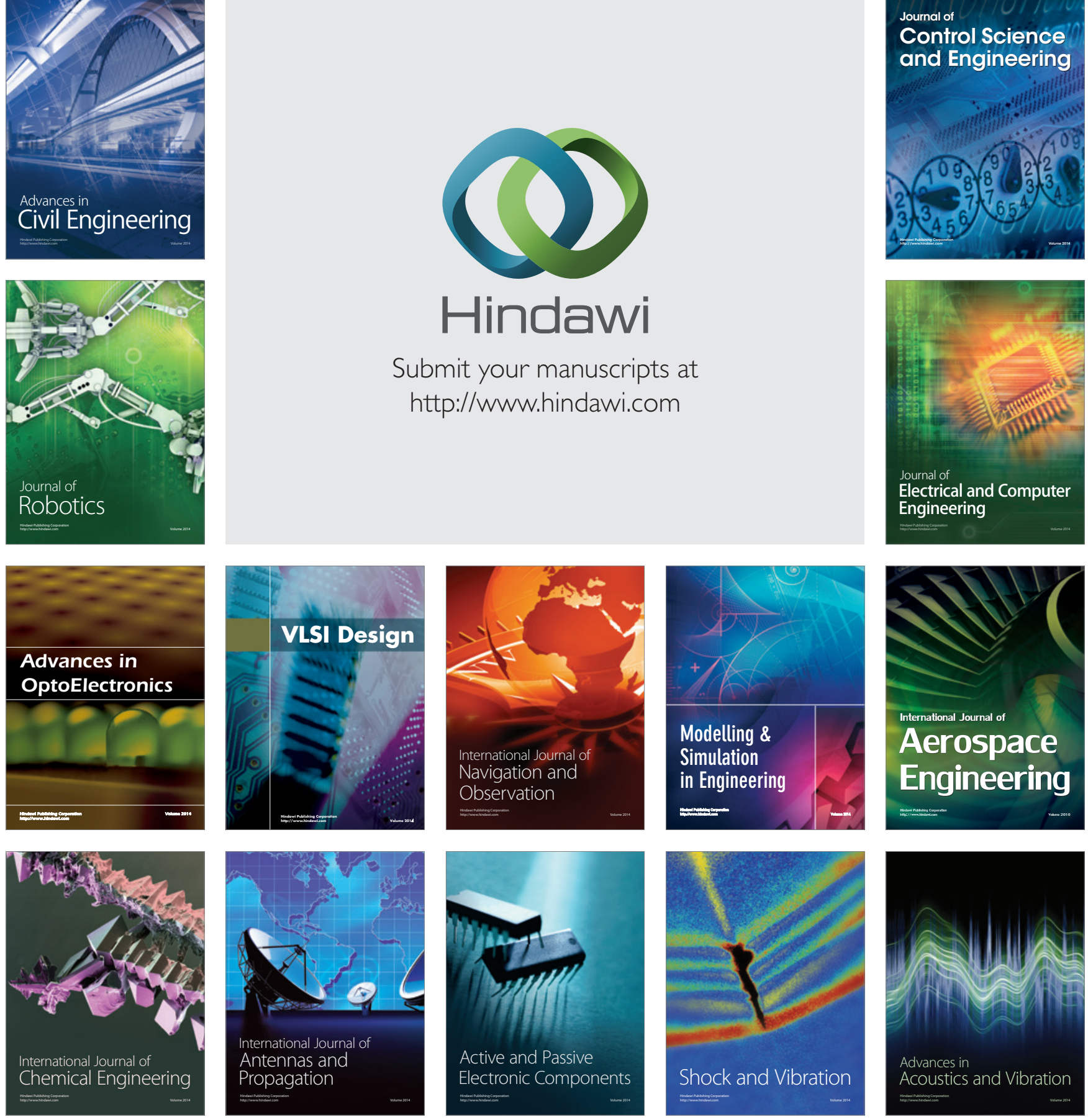